21st Particles and Nuclei International Conference (PANIC 2017)

International Journal of Modern Physics: Conference Series

Vol. 46 (2018) 1860050 (6 pages)

(C) The Author(s)

DOI: $10.1142 / \mathrm{S} 2010194518600509$

\title{
The Role of the Isospin 3/2 Component in Elastic Neutron-Deuteron Scattering and in the Deuteron Breakup Reaction
}

\author{
H. Witała*, J. Golak, R. Skibiński and K. Topolnicki \\ M. Smoluchowski Institute of Physics, \\ Jagiellonian University, PL-30348 Kraków, Poland \\ *henryk.witala@uj.edu.pl \\ H. Kamada \\ Department of Physics, Faculty of Engineering, \\ Kyushu Institute of Technology, Kitakyushu 804-8550, Japan
}

Published 3 May 2018

\begin{abstract}
We discuss the importance of the three-nucleon isospin $T=3 / 2$ component in elastic neutron-deuteron scattering and in the deuteron breakup reaction. The contribution of this amplitude originates from charge-independence breaking of the nucleon-nucleon potential. We study the magnitude of that contribution to the elastic scattering and breakup observables, taking the Av18 nucleon-nucleon potential alone or combined with the Urbana IX three-nucleon force as well as the locally regularized chiral $\mathrm{N}^{4} \mathrm{LO}$ nucleonnucleon potential alone or supplemented by the chiral $\mathrm{N}^{2} \mathrm{LO}$ three-nucleon force. We find that the isospin $T=3 / 2$ component is important for the breakup reaction and the proper treatment of charge-independence breaking in this case requires the inclusion of the ${ }^{1} S_{0}$ state with isospin $T=3 / 2$. For neutron-deuteron elastic scattering the $T=3 / 2$ contributions are insignificant and charge-independence breaking can be accounted for by neglecting $T=3 / 2$ component and using the effective t-matrix generated with the so-called " $2 / 3-1 / 3$ " rule.
\end{abstract}

Keywords: Three-nucleon reactions; nucleon-nucleon potential; Faddeev equation.

\section{Introduction}

Charge-independence breaking (CIB) is well established in the two-nucleon $(2 \mathrm{~N})$ system as evidenced e.g. by the values of the ${ }^{1} S_{0}$ scattering lengths, different for the neutron-proton (np), proton-proton ( $\mathrm{pp}$ ) (with the Coulomb force subtracted), and neutron-neutron (nn) systems. Modern, high precision NN potentials, such as the standard semi-phenomenological model $\mathrm{Av}^{1} 8^{1}$ as well as the chiral $\mathrm{NN}$ forces ${ }^{2}$

This is an Open Access article published by World Scientific Publishing Company. It is distributed under the terms of the Creative Commons Attribution 4.0 (CC-BY) License. Further distribution of this work is permitted, provided the original work is properly cited. 


\section{H. Witata et al.}

incorporate the present knowledge of CIB. The isospin violating $2 \mathrm{~N}$ forces induce an admixture of the total isospin $T=3 / 2$ on top of the dominant $T=1 / 2$ state in the three-nucleon $(3 \mathrm{~N})$ system. The CIB of the NN interaction thus affects $3 \mathrm{~N}$ observables. The detailed treatment of the $3 \mathrm{~N}$ system with CIB NN forces in the case of distinguishable or identical particles was formulated and described in. ${ }^{3}$ To investigate the role of the amplitude with the total three-nucleon $(3 \mathrm{~N})$ isospin $T=3 / 2$ in elastic neutron-deuteron (nd) scattering and in the deuteron breakup reaction and to check if conclusions depend on the employed dynamics we performed calculations of these processes using two different dynamical inputs. Taking the standard semi-phenomenological potential Av18 we applied it alone or combined with the UrbanaIX (UIX) ${ }^{4} 3 \mathrm{NF}$, and when using the recent chiral $\mathrm{NN}$ interaction ${ }^{5}$ we applied it alone or augmented by the chiral $\mathrm{N}^{2} \mathrm{LO} 3 \mathrm{~N}$ force. ${ }^{6}$ In Sec. 2 we briefly describe the formalism of $3 \mathrm{~N}$ continuum Faddeev calculations and the inclusion of CIB. The results are presented in Sec. 3. We summarize and conclude in Sec. 4.

\section{2. $3 \mathrm{~N}$ scattering and charge independence breaking}

Neutron-deuteron scattering with nucleons interacting through a NN interaction $v_{N N}$ and a $3 \mathrm{NF} V_{123}$, is described in terms of a breakup operator $T$ satisfying the Faddeev-type integral equation ${ }^{7,8}$

$$
\begin{aligned}
T|\phi\rangle= & t P|\phi\rangle+\left(1+t G_{0}\right) V^{(1)}(1+P)|\phi\rangle+t P G_{0} T|\phi\rangle \\
& +\left(1+t G_{0}\right) V^{(1)}(1+P) G_{0} T|\phi\rangle .
\end{aligned}
$$

The two-nucleon $t$-matrix $t$ is the solution of the Lippmann-Schwinger equation with the interaction $v_{N N} . V^{(1)}$ is the part of a $3 \mathrm{NF}$ which is symmetric under the interchange of nucleons 2 and $3: V_{123}=V^{(1)}(1+P)$. The permutation operator $P=P_{12} P_{23}+P_{13} P_{23}$ is given in terms of the transposition operators, $P_{i j}$, which interchange nucleons $i$ and $j$. The incoming state $|\phi\rangle=\left|\mathbf{q}_{0}\right\rangle\left|\phi_{d}\right\rangle$ describes the free relative motion of the neutron and the deuteron with the relative momentum $\mathbf{q}_{0}$ and contains the internal deuteron state $\left|\phi_{d}\right\rangle$. Finally, $G_{0}$ is the resolvent of the threebody center-of-mass kinetic energy. The amplitude for elastic scattering leading to the corresponding two-body final state $\left|\phi^{\prime}\right\rangle$ is then given by ${ }^{7,8}$

$$
\begin{aligned}
\left\langle\phi^{\prime}|U| \phi\right\rangle= & \left\langle\phi^{\prime}\left|P G_{0}^{-1}\right| \phi\right\rangle+\left\langle\phi^{\prime}|P T| \phi\right\rangle+\left\langle\phi^{\prime}\left|V^{(1)}(1+P)\right| \phi\right\rangle \\
& +\left\langle\phi^{\prime}\left|V^{(1)}(1+P) G_{0} T\right| \phi\right\rangle,
\end{aligned}
$$

while for the breakup reaction one has

$$
\left\langle\phi_{0}^{\prime}\left|U_{0}\right| \phi\right\rangle=\left\langle\phi_{0}^{\prime}|(1+P) T| \phi\right\rangle
$$

where $\left|\phi_{0}^{\prime}\right\rangle$ is the free three-body breakup channel state.

Solving Eq.(1) in the momentum-space partial wave basis, defined by the magnitudes of the $3 \mathrm{~N}$ Jacobi momenta $p$ and $q$ together with the angular momenta and isospin quantum numbers $\alpha(\beta)$, is performed by projecting Eq. (1) onto two 
types of basis states: $|p q \alpha\rangle \equiv \mid p q$ angular momenta $\rangle\left|\left(t \frac{1}{2}\right) T=\frac{1}{2} M_{T}\right\rangle,(t=0,1)$, and $|p q \beta\rangle \equiv \mid p q$ angular momenta $\rangle\left|\left(t \frac{1}{2}\right) T=\frac{3}{2} M_{T}\right\rangle,(t=1)$. The $2 \mathrm{~N}$ t-operator in the three-particle isospin space can be written for the nd system $\operatorname{as}^{3}$ (we assume that the neutron (proton) isospin projection is $\left.\frac{1}{2}\left(-\frac{1}{2}\right)\right)$ :

$$
\begin{aligned}
& \left\langle\left(t \frac{1}{2}\right) T M_{T}=\frac{1}{2}|t|\left(t^{\prime} \frac{1}{2}\right) T^{\prime} M_{T^{\prime}}=\frac{1}{2}\right\rangle=\delta_{t t^{\prime}} \delta_{T T^{\prime}} \delta_{T 1 / 2}\left[\delta_{t 0} t_{n p}^{t=0}+\delta_{t 1}\left(\frac{2}{3} t_{n n}^{t=1}+\frac{1}{3} t_{n p}^{t=1}\right)\right] \\
& +\delta_{t t^{\prime}} \delta_{t 1}\left(1-\delta_{T T^{\prime}}\right) \frac{\sqrt{2}}{3}\left(t_{n n}^{t=1}-t_{n p}^{t=1}\right)+\delta_{t t^{\prime}} \delta_{t 1} \delta_{T T^{\prime}} \delta_{T 3 / 2}\left(\frac{1}{3} t_{n n}^{t=1}+\frac{2}{3} t_{n p}^{t=1}\right),
\end{aligned}
$$

where $t_{n n}$ and $t_{n p}$ are the $n n$ and np t-matrices, respectively.

As a result of solving Eq. (1) one gets the amplitudes $\langle p q \alpha|T| \phi\rangle$ and $\langle p q \beta|T| \phi\rangle$, which fulfill the following set of coupled integral equations:

$$
\begin{aligned}
& \langle p q \alpha|T| \phi\rangle=\sum_{\alpha^{\prime}} \int_{p^{\prime} q^{\prime}}\left\langle p q \alpha|t| p^{\prime} q^{\prime} \alpha^{\prime}\right\rangle\left\langle p^{\prime} q^{\prime} \alpha^{\prime}|P| \phi\right\rangle+\sum_{\alpha^{\prime}} \int_{p^{\prime} q^{\prime}}\left\langle p q \alpha\left|V^{(1)}\right| p^{\prime} q^{\prime} \alpha^{\prime}\right\rangle \\
\times & \left\langle p^{\prime} q^{\prime} \alpha^{\prime}|(1+P)| \phi\right\rangle+\sum_{\alpha^{\prime}} \int_{p^{\prime} q^{\prime}}\left\langle p q \alpha|t| p^{\prime} q^{\prime} \alpha^{\prime}\right\rangle\left\langle p^{\prime} q^{\prime} \alpha^{\prime}\left|G_{0} V^{(1)}(1+P)\right| \phi\right\rangle \\
+ & \sum_{\alpha^{\prime}} \int_{p^{\prime} q^{\prime}}\left\langle p q \alpha|t| p^{\prime} q^{\prime} \alpha^{\prime}\right\rangle\left\langle p^{\prime} q^{\prime} \alpha^{\prime}\left|P G_{0} T\right| \phi\right\rangle+\sum_{\beta^{\prime}} \int_{p^{\prime} q^{\prime}}\left\langle p q \alpha|t| p^{\prime} q^{\prime} \beta^{\prime}\right\rangle\left\langle p^{\prime} q^{\prime} \beta^{\prime}\left|P G_{0} T\right| \phi\right\rangle \\
+ & \sum_{\alpha^{\prime}} \int_{p^{\prime} q^{\prime}}\left\langle p q \alpha\left|V^{(1)}\right| p^{\prime} q^{\prime} \alpha^{\prime}\right\rangle\left\langle p^{\prime} q^{\prime} \alpha^{\prime}\left|(1+P) G_{0} T\right| \phi\right\rangle+\sum_{\alpha^{\prime}} \int_{p^{\prime} q^{\prime}} \sum_{\alpha^{\prime \prime}} \int_{p^{\prime \prime} q^{\prime \prime}}\left\langle p q \alpha|t| p^{\prime} q^{\prime} \alpha^{\prime}\right\rangle \\
\times & \left\langle p^{\prime} q^{\prime} \alpha^{\prime}\left|G_{0} V^{(1)}\right| p^{\prime \prime} q^{\prime \prime} \alpha^{\prime \prime}\right\rangle\left\langle p^{\prime \prime} q^{\prime \prime} \alpha^{\prime \prime}\left|(1+P) G_{0} T\right| \phi\right\rangle+\sum_{\beta^{\prime}} \int_{p^{\prime} q^{\prime}} \sum_{\beta^{\prime \prime}} \int_{p^{\prime \prime} q^{\prime \prime}}\left\langle p q \alpha|t| p^{\prime} q^{\prime} \beta^{\prime}\right\rangle \\
\times & \left\langle p^{\prime} q^{\prime} \beta^{\prime}\left|G_{0} V^{(1)}\right| p^{\prime \prime} q^{\prime \prime} \beta^{\prime \prime}\right\rangle\left\langle p^{\prime \prime} q^{\prime \prime} \beta^{\prime \prime}\left|(1+P) G_{0} T\right| \phi\right\rangle, \sum_{\alpha^{\prime}} \int_{p^{\prime} q^{\prime}}\left\langle p q \beta|t| p^{\prime} q^{\prime} \alpha^{\prime}\right\rangle\left\langle p^{\prime} q^{\prime} \alpha^{\prime}|P| \phi\right\rangle+\sum_{\alpha^{\prime}} \int_{p^{\prime} q^{\prime}}\left\langle p q \beta|t| p^{\prime} q^{\prime} \alpha^{\prime}\right\rangle \\
& \langle p q \beta|T| \phi\rangle=\sum_{\beta^{\prime}}(5) \\
\times & \left\langle p^{\prime} q^{\prime} \alpha^{\prime}\left|G_{0} V^{(1)}(1+P)\right| \phi\right\rangle+\sum_{\alpha^{\prime}} \int_{p^{\prime} q^{\prime}}\left\langle p q \beta|t| p^{\prime} q^{\prime} \alpha^{\prime}\right\rangle\left\langle p^{\prime} q^{\prime} \alpha^{\prime}\left|P G_{0} T\right| \phi\right\rangle \\
+ & \sum_{\beta^{\prime}} \int_{p^{\prime} q^{\prime}}\left\langle p q \beta|t| p^{\prime} q^{\prime} \beta^{\prime}\right\rangle\left\langle p^{\prime} q^{\prime} \beta^{\prime}\left|P G_{0} T\right| \phi\right\rangle+\sum_{\beta^{\prime}} \int_{p^{\prime} q^{\prime}}\left\langle p q \beta\left|V^{(1)}\right| p^{\prime} q^{\prime} \beta^{\prime}\right\rangle \\
\times & \left\langle p^{\prime} q^{\prime} \beta^{\prime}\left|(1+P) G_{0} T\right| \phi\right\rangle+\sum_{\alpha^{\prime}} \int_{p^{\prime} q^{\prime}} \sum_{\alpha^{\prime \prime}} \int_{p^{\prime \prime} q^{\prime \prime}}\left\langle p q \beta|t| p^{\prime} q^{\prime} \alpha^{\prime}\right\rangle\left\langle p^{\prime} q^{\prime} \alpha^{\prime}\left|G_{0} V^{(1)}\right| p^{\prime \prime} q^{\prime \prime} \alpha^{\prime \prime}\right\rangle \\
\times & \left\langle p^{\prime \prime} q^{\prime \prime} \alpha^{\prime \prime}\left|(1+P) G_{0} T\right| \phi\right\rangle+\sum_{\beta^{\prime} q^{\prime}} \sum_{\beta^{\prime \prime}}\left\langle p q \beta|t| p^{\prime} q^{\prime} \beta^{\prime}\right\rangle\left\langle p^{\prime} q^{\prime} \beta^{\prime}\left|G_{0} V^{\prime \prime}{ }^{\prime 1}\right| p^{\prime \prime} \mid(1+P) q_{0}^{\prime \prime} \beta^{\prime \prime}\right\rangle \\
& \langle\phi\rangle .
\end{aligned}
$$

The form of the couplings in Eq. (5) follows from the fact that the incoming neutrondeuteron state $|\phi\rangle$ has the total isospin $T=1 / 2$, the permutation operator $P$ is diagonal in the total isospin, and the $3 \mathrm{NF}$ is assumed to conserve the total isospin $T$.

From Eqs. (5) it is clear that the amplitudes $\langle p q \beta|T| \phi\rangle$ will be nonzero only when the $n n$ and $n p$ interactions differ in the same orbital and spin angular-momentum 


\section{H. Witata et al.}

states with the $2 \mathrm{~N}$ subsystem isospin $t=1$ (CIB). It is the set of equations (5) which we solve when we differentiate between the $\mathrm{nn}$ and $\mathrm{np}$ interactions and include both $T=1 / 2$ and $T=3 / 23 \mathrm{NF}$ matrix elements. In the case when one neglects the $T=3 / 2$ amplitudes, the CIB can be approximately taken care of by using the effective two-body t-matrix: $t_{\text {eff }}=\frac{2}{3} t_{n n}^{t=1}+\frac{1}{3} t_{n p}^{t=1}$, generated with the so-called " $2 / 3-1 / 3$ " rule in the $2 \mathrm{~N}$ subsystem isospin $t=1$ states (see Eq.(4)).

Since the final state $\left|\phi^{\prime}\right\rangle$ in elastic nd scattering has also the total $3 \mathrm{~N}$ isospin $T=1 / 2$, the amplitudes $\langle p q \beta|T| \phi\rangle$ do not contribute directly to this reaction. However, the $T=3 / 2$ admixture enters in this case through a modification of the $T=1 / 2$ amplitudes $\langle p q \alpha|T| \phi\rangle$ induced by the couplings given in Eqs. (5). Contrary to that, for the nd breakup reaction both $T=1 / 2$ and $T=3 / 2$ amplitudes contribute significantly.

\section{Results}

We solved the $3 \mathrm{~N}$ Faddeev equations (5) at four values of the incoming neutron laboratory energy: $E_{l a b}=13,65,135$, and $250 \mathrm{MeV} .{ }^{9}$ To check that the conclusions do not depend on the dynamical input used we took as a NN potential the Av18 interaction alone or combined with the Urbana IX $3 \mathrm{NF}$ as well as the semilocally regularized $\mathrm{N}^{4} \mathrm{LO}$ chiral potential of ref. ${ }^{5,10}$ with the regulator $R=0.9 \mathrm{fm}$, alone or supplemented with the chiral $\mathrm{N}^{2} \mathrm{LO} 3 \mathrm{NF},{ }^{6}$ regularized with the same regulator. As a nn force we took the pp versions of these particular NN interactions (with the Coulomb force subtracted). In order to provide convergent predictions we solved Eqs. (5) taking into account all partial wave states with the total $2 \mathrm{~N}$ angular momenta up to $j_{\max }=5$ and $3 \mathrm{~N}$ total angular momenta up to $J_{\max }=25 / 2$. The $3 \mathrm{NF}$ was included up to $J_{\max }=7 / 2$.

Obtained results for the total nd scattering cross section, the total nd elastic scattering cross section, and the total nd breakup cross section show that it is sufficient to use the effective t-matrix $t_{\text {eff }}$ and to neglect $T=3 / 2$ states completely to account exactly for CIB effects in all three total cross sections. That is true both when the $3 \mathrm{NF}$ is included or omitted. The exact treatment of CIB by using in all $t=1$ states the $t_{n p}$ and $t_{n n}$ t-matrices and both $T=1 / 2$ and $T=3 / 2$ partial wave states as well as restricting the exact treatment of CIB to the ${ }^{1} S_{0}$ state provide just the same values for the total cross sections. It shows that contribution of $T=3 / 2$ states, as far as the total cross sections are concerned, can be neglected.

As in the case of the total cross sections also for exclusive elastic nd scattering the three treatments of the CIB, namely using $t_{\text {eff }}$ and no $T=3 / 2$ states, $T=3 / 2$ in the ${ }^{1} S_{0}$ state with the $t_{n p}$ and $t_{n n}$ t-matrices in that state, and $T=3 / 2$ in all $t=1$ states with the corresponding $\mathrm{np}$ and $\mathrm{nn}$ t-matrices, provide the same elastic differential cross sections. We checked that also for all nd elastic scattering spin observables, encompassing the neutron (vector) and deuteron (vector and tensor) analyzing powers, the spin correlation as well as spin transfer coefficients, the above mentioned three approaches lead to the same results. Thus again the contribution 
of all $T=3 / 2$ states can be neglected and CIB in elastic nd scattering treated exactly by restricting the calculation to $T=1 / 2$ states and using in all $t=1$ states the " $2 / 3-1 / 3$ " rule to generate from $t_{n p}$ and $t_{n n}$ the effective $t_{\text {eff }}$ t-matrix.

In the final exclusive breakup state both $T=1 / 2$ and $T=3 / 2$ total isospin components are allowed. Thus one would expect that here the influence of the $T=3 / 2$ components will be better visible than in elastic scattering. Our results show that this is indeed the case in a large region of the breakup phase-space. That component is especially important in kinematically complete final-state-interaction (FSI) breakup configurations. In the FSI configuration under the exact FSI condition, the two outgoing nucleons have equal momenta. Their strong interaction in the ${ }^{1} S_{0}$ state leads to a characteristic cross section maximum, the magnitude of which is sensitive to the ${ }^{1} S_{0}$ scattering length. Since largest CIB effects are seen in the difference between $\mathrm{np}$ and $\mathrm{nn}(\mathrm{pp})^{1} S_{0}$ scattering lengths, the region of the FSI peak reveals largest CIB effects. That is demonstrated in Fig. 1 for the cross sections in the $n n$ and np FSI configurations. It is seen that restricting the calculation to $t_{\text {eff }}$ only and neglecting the $T=3 / 2$ components is insufficient to include all CIB effects. Inclusion of the $T=3 / 2$ component in the ${ }^{1} S_{0}$ state only is, however, sufficient to fully account for the CIB effects. This shows that the proper treatment of CIB in the FSI configurations of the nd breakup requires the inclusion of the total isospin $T=3 / 2$ component and using both $t_{n p}$ and $t_{n n}$ t-matrices just in the ${ }^{1} S_{0}$ state.

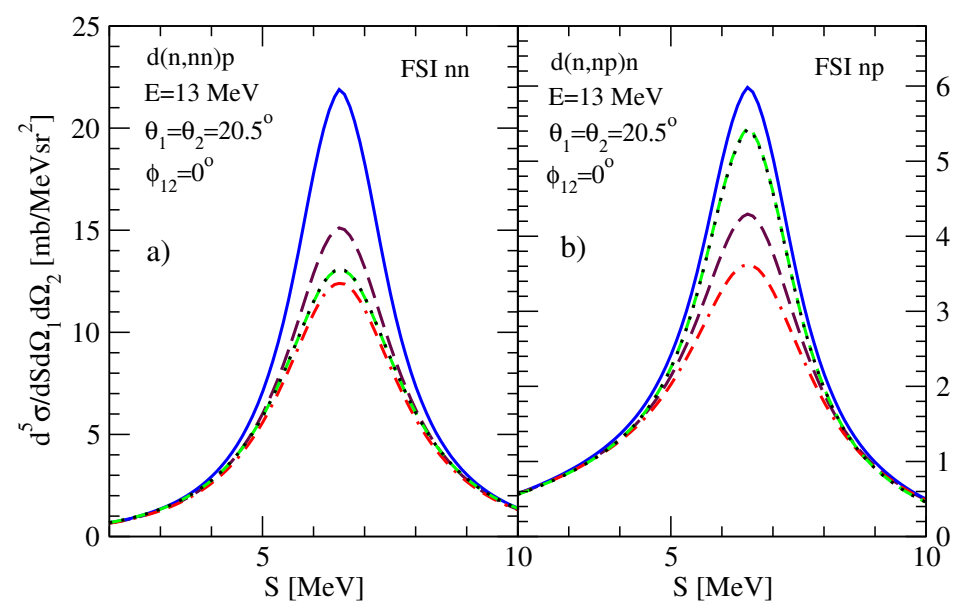

Fig. 1. (color online) The cross section $d^{5} \sigma / d \Omega_{1} d \Omega_{2} d S$ for the nn (a) and np (b) FSI configuration as a function of the arc-length S. Different lines are predictions obtained with the Av18 NN potential alone and different underlying dynamics: long-dashed (maroon) line - in all $t=1$ states effective t-matrix $t_{\text {eff }}$, solid (blue) line - in the ${ }^{1} S_{0}$ state np force and in all other $t=1$ states the effective t-matrix $t_{\text {eff }}$, double-dashed-dotted (red) line - in the ${ }^{1} S_{0}$ state the nn force and in all other $t=1$ states the effective t-matrix $t_{\mathrm{eff}}$, short-dashed (green) line - in the ${ }^{1} S_{0}$ channels $\mathrm{np}$ and $\mathrm{nn}$ forces with the isospin $T=3 / 2$ included and in all other $t=1$ states the effective t-matrix $t_{\text {eff }}$, dotted (black) line - in all $t=1$ states $n p$ and $n n$ forces and the isospin $T=3 / 2$ included. 


\section{H. Witata et al.}

\section{Summary}

We investigated the importance of the scattering amplitude components with the total $3 \mathrm{~N}$ isospin $T=3 / 2$ in elastic nd scattering and in the deuteron breakup reaction. In general, the inclusion of these components is required to account for CIB effects of the NN interaction. The modern NN interactions, which describe existing $\mathrm{pp}$ and $\mathrm{np}$ data with high precision, provide $\mathrm{pp}$ and $\mathrm{np}$ t-matrices which differ up to $\approx 10 \%$. Such a magnitude of CIB requires that the isospin $T=3 / 2$ components are included in the calculation of the breakup reaction, especially for the regions of the breakup phase-space close to the FSI condition. However, in order to account for all CIB effects it is sufficient to restrict the inclusion of $T=3 / 2$ to the ${ }^{1} S_{0}$ partial wave state only instead of doing it in all $t=1$ states. For elastic scattering we found that the $T=3 / 2$ components can be neglected completely and all CIB effects accounted for by restricting calculations to total $3 \mathrm{~N}$ isospin $T=1 / 2$ partial waves only and using the effective t-matrix generated with the " $2 / 3-1 / 3^{\prime \prime}$ rule: $t_{\mathrm{eff}}=(2 / 3) t_{n n}+(1 / 3) t_{n p}$. The obtained results show that in nd reactions the $T=3 / 2$ component is overshadowed by the dominant $T=1 / 2$ contribution.

\section{Acknowledgments}

This work was performed with support from the Polish National Science Center under Grant No's. 2016/22/M/ST2/00173 and 2016/21/D/ST2/01120. The numerical calculations have been performed on the supercomputer cluster of the JSC, Jülich, Germany.

\section{References}

1. R. B. Wiringa, V. G. J. Stoks, R. Schiavilla, Phys. Rev. C51, 38 (1995).

2. E. Epelbaum, Prog. Part. Nuclear Phys. 57, 654 (2006).

3. H. Witała, W. Glöckle and H. Kamada, Phys. Rev. C43, 1619 (1991).

4. B. S. Pudliner et al., Phys. Rev. C56, 1720 (1997).

5. E. Epelbaum, H. Krebs and Ulf-G. Meißner, Eur. Phys. J. A51, 53 (2015).

6. E. Epelbaum et al., Phys. Rev. C66, 064001 (2002).

7. W. Glöckle, H. Witała, D. Hüber, H. Kamada, J. Golak, Phys. Rep. 274, 107 (1996).

8. D. Hüber et al., Acta Physica Polonica B28, 1677 (1997).

9. H. Witała et al., Pew-Body Syst. 57, 1213 (2016).

10. S. Binder et al., Phys. Rev. C93, 044002 (2016). 